No. 11(18)

\title{
VORTICES \\ AND COMPLEX NUMBERS
}

\section{Arkadiusz Maciuk, Antoni Smoluk}

\begin{abstract}
The paper emphasizes that complex numbers are objects with their equivalents commonly occurring in nature. Just like real numbers measure lengths in a physical world, complex numbers measure vortices observed in nature. The spiral orbits in this paper are exponential spirals (also called logarithmic spirals). A vortex is identified by determining a complex number that generates it. To determine this number, we need two snap-reading observations that provide the argument of a complex number, while the ratio of radiuses the modulus of a complex number. Therefore, we also deal with the area of a complex number. Complex numbers involve a meaningful description of the laws of nature, i.e. of vortices and of equilibrium.
\end{abstract}

Keywords: complex number, vortex.

JEL Classification: C02, C60.

DOI: $10.15611 / \mathrm{me} .2015 .11 .07$.

\section{Introduction}

All number systems originate from an additive semigroup of natural numbers $\mathbb{N}$. The simplest extension of this semigroup is $\mathbb{Z}-$ a ring of integer numbers. Its further extension without the ring that has no zero divisors is $\mathbb{Q}$ - a field of rational numbers. A continuous extension of a field is the set of real numbers $\mathbb{R}$, typically thought of as points on a straight line. Natural numbers serve as counters of discrete objects, integer numbers assist in comparing discrete sets: more or less, and how much more or less, while rational numbers help to divide a whole into parts, and real numbers - to measure distances. Hence, each number system is destined for some practical application. Most people are satisfied with those practical number systems because complex numbers, once called imaginary numbers, are regarded as a strange idea of a mathematician who created it merely to have a new object of interest. However, nobody has any doubts that in nature there exist disks such as round cakes and the circular sectors of such

\footnotetext{
Arkadiusz Maciuk, Antoni Smoluk

Department of Mathematics and Cybernetics, Wrocław University of Economics

arkadiusz.maciuk@ue.wroc.pl
} 
disks - slices of cakes. These portions of cake shapes that we call here circular triangles represent precisely complex numbers. Complex numbers are not pure inventions of unworldly mathematicians, but more practical and ordinary than real numbers. The most frequent definition of a complex number is equivalent to a pair of real numbers. Hence, a set of complex numbers $\mathbb{C}$ is frequently, and for a very good reason, identified with a plane $\mathbb{R}^{2}$, i.e. a Cartesian product of two straight lines. Writing a complex number $c \in \mathbb{C}$ in the form $z=a+b i$ actually means that $z=(a, b)=a(1,0)+b(0,1)$. The vector $(1,0)$ is treated as a unit in the field of complex numbers, while the vector $i=(0,1)$ is called a complex unit. A linear algebra $\mathbb{C}$ of dimension 2 over a field $\mathbb{R}$ becomes a field of complex numbers once we assume one relation defining that $i^{2}=-(1,0)$.

The idea of a circle commonly occurring in nature can be derived from a field of real numbers. Namely, a circle $\mathbb{T}$ is a quotient group of a field of real numbers $\mathbb{R}$ by a subgroup $2 \pi \mathbb{Z}$ of integer multiples of a number $2 \pi$. Thus, it holds

$$
\mathbb{T}=\mathbb{R} / 2 \pi \mathbb{Z} .
$$

\section{Archimedes and complex numbers}

A circle implies angles, therefore angles are equivalence classes of real numbers translated by $2 \pi n$, where $n \in \mathbb{N}$. Archimedes' constant $\pi$ used to define a circle has merely a normalizing character that comes from the claim that the circumference of a circle with the radius 1 equals $2 \pi$. Since $\mathrm{Ar}$ chimedes introduced the notion of an arc whose essence is an angle (Figure 1 ), hence he can be regarded as the pioneer and even as the father of complex numbers. He did not invent them, but discovered them - as they exist in nature as circular objects such as trees, stones, shapes of planets and their orbits. Complex numbers can be interpreted as linear transformations of two-dimensional space $\mathbb{R}^{2}$. Linear map $\left(\begin{array}{cc}a & -b \\ b & a\end{array}\right)$ is an analogue of complex number $z=a+b i$. The equality of isomorphic objects does not imply their sameness, but their correspondence, equivalence. This isomorphism identifies complex numbers with orthogonal transformations of a given shape; orthogonality here means that rows are orthogonal vectors. If the radius of a complex number $r=\sqrt{a^{2}+b^{2}}$ is strictly greater than 1 , then the 
above-given linear operation is the composition of rotation by an angle represented by $z$ and of dilation represented by the radius $r$. On the other hand, if $r$ is strictly less than 1 , then the linear operation is reduced to a contraction, i.e. a shrinkage. The equality of a unitary radius denotes a pure rotation. A dilation implies that successive compositions of linear operations represented by $r$ diverge from the point of equilibrium, whereas the radius that is less than unity secures the convergence to the point of equilibrium. All vortices observed in the plane, such as flowing water or windswept leaves, have this nature. Thus, complex numbers can be observed in nature because they model what we typically see, i.e. the states of equilibrium or its perturbation - shifting away from equilibrium.

\section{Isomorphic models of complex numbers}

A multiplicative group $\mathbb{C}^{*}$ of nonzero complex numbers is isomorphic to a product group $\mathbb{T} \times \mathbb{R}_{+}^{*}$, where $\mathbb{R}_{+}^{*}$ denotes a multiplicative group of real positive numbers. Thus, each complex number is a pair: an angle and a radius (Figure 1 ). So it holds $z=r u$, where $r \in \mathbb{R}_{+}$is a radius, while $u$ belonging to a group $\mathbb{T}$ is identical to an angle $\phi$ since it holds $u=\cos \phi+i \sin \phi, 0 \leq \phi<2 \pi$. In this paper, isomorphic structures are regarded as identical by default - they are not distinguished.

A complex number therefore is a circular triangle with the area $\sigma=\frac{1}{2} r \alpha$, where $\alpha$ is an arc of the length $r \phi$. Hence the area of a circular triangle equals $\sigma=\frac{1}{2} r^{2} \phi$. Only those complex numbers that are also real, strictly positive numbers have the zero area. Numbers with a zero imaginary part, i.e. those with $\phi=0$, have the zero area, as they are line segments. Complex numbers are also bivectors; the number $z=a+b i$ is represented by a pair of orthogonal vectors $(a, b),(-b, a)$ (Figure 2$)$. These vectors determine a square whose area is radius squared, i.e. $S=r^{2}=a^{2}+b^{2}$. In the latter case each non-zero complex number has a strictly positive area. Only the number zero has a zero area. A twofold emphasis on the area of a complex number indicates that these numbers are flat entities - they represent angles and arcs. The modulus of a complex number therefore is a root of its area $S$. 


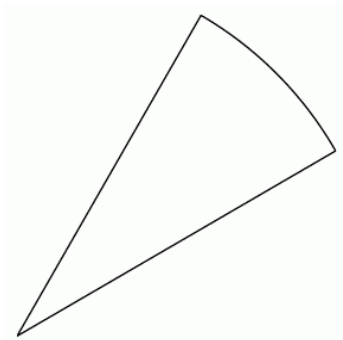

Fig. 1. A complex number - a piece of cake

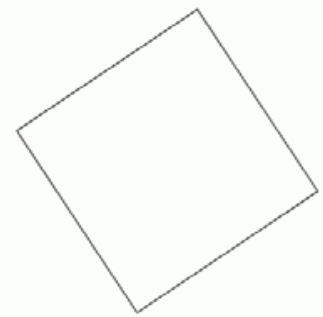

Fig. 2. Bivector - a complex number

Source: own elaboration.

Complex numbers are entities represented by arcs and circles or by linear transformations being actually bivectors - squares. So, a complex number can be interpreted as a circular triangle - a piece of cake, or as a square - a linear transformation. These are tangible entities, at least to a degree like real numbers are tangible. Real numbers measure just lengths, while complex numbers measure both lengths and arcs, hence they are said to have an area.

\section{Vortices and spirals}

A subgroup $\mathbb{T}_{n}$ of $n$th roots of unity of a group $\mathbb{T}$ can be identified with a gear. A set of such subgroups represents a mechanical clock. These subgroups contain cyclicality of time. Following a number of rotations the cogs of a gear mesh together like they did at the beginning. Periodic functions occur in nature in the same way as complex numbers; complex numbers and periodic functions model phenomena called vortices. What is a periodic function? Each function whose domain is the circle group $\mathbb{T}$ is said to be periodic. The most important periodic function is the cosine and other trigonometric functions naturally related with the cosine. The idea of periodicity is fundamental in natural sciences. Life can be defined as the continuous renovation of an organism. Linear maps

$$
A(z)=A z, \quad \text { where } \quad A=\left(\begin{array}{cc}
a & -b \\
b & a
\end{array}\right)
$$

are a matrix representation of a complex number $a+i b$, in a complex domain are reduced to composition of rotations and real linear functions. Rotation is the essence of periodicity. 
If matrix $A \neq 0$, then a group of linear transformations $A_{t}(z)=(a+b i)^{t} u$, with a composition satisfying a natural condition $A_{t_{1}}^{\circ} A_{t_{2}}=A_{t_{1}+t_{2}}, t \in \mathbb{R}, u \in \mathbb{C}$, is connected with the above linear operation. As a consequence, a group of operators generated by matrix $\boldsymbol{A}$ is obtained. An orbit of the point $u \in \mathbb{C}$ is the set $O(u)=\left\{A_{t}(u): t \in \mathbb{R}\right\}$ that is a spiral function of the parameter $t$ with equation $r_{A}(t)=\rho^{t} e^{(\psi+t \phi) i}$, where $A=\rho e^{i \phi}, u=e^{i \psi}$ and $t$ denotes time. Exactly one orbit passes through every point of the group $\mathbb{C}^{*}$. An orbit is a subset of a field $\mathbb{C}$. A function $f$ specifying the orbit $O(u)$ is a subset of $\mathbb{R} \times \mathbb{C}$, because $f_{u}=\left\{\left(t, A^{t} u\right): t \in \mathbb{R}\right\}$, thus $f_{u}(t)=A^{t} u$. A function $f_{u}$ is the trajectory of the point $u$ moving along the orbit $O(u)$. In order to obtain all possible orbits, it suffices to consider only the numbers belonging to the circle group $\mathbb{T}$. A vortex of the number $A$ denoted by $\operatorname{Vor}(A)$ is a family of orbits $\operatorname{Vor}(A)=\{O(u): u \in T\}$. Obviously, orbits of different points in the circle $\mathbb{T}$ are disjointed. The orbits of points in the circle with the radius $\rho \in \mathbb{R}_{+}^{*}$ are formed by the orbits of points in the circle $\mathbb{T}$ : it suffices to multiply the latter by $\rho$. The scale is changed; the radius $\rho$ becomes a unity.
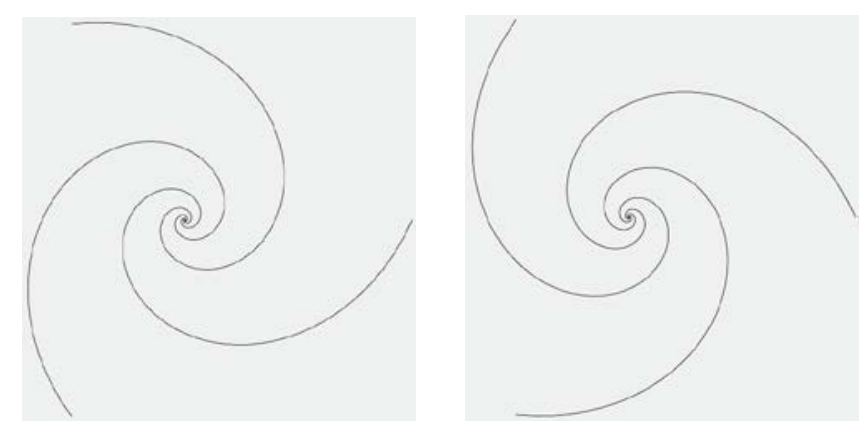

Fig. 3. Vortices generated by the number $2 i$ and the number $-2 i$ demonstrated respectively by three orbits of points being the cube roots of 1

Source: own elaboration.

A family of spirals $\left\{r_{A}: A \in \mathbb{C}^{*}\right\}$ is the image of the vortex of the number $A$ (Figure 3). A spiral $O(u)$ is the orbit of the point $u \in \mathbb{C}^{*}$, the image 
of a half-line $z=t u, t \in \mathbb{R}_{+}^{*}$, using the isomorphism of the additive group $\mathbb{R}$ and the multiplicative group $\mathbb{R}_{+}^{*}$ The image of a circle centered at zero is also a circle with a center at zero, with a suitably modified radius when $|A| \neq 1$. If a modulus of $A$ is greater than 1 , then spirals diverge; if it is equal to 1 , then the motion is purely circular; when a modulus of $A$ is less than 1 , spirals converge in the point 0 . Spiral orbits are actually exponential spirals with an equation in polar coordinates $r=a e^{b t}$ where $t \in \mathbb{R}$.

The angle $\phi$ of the number $A$ determines the intensity of convolutions and the direction of the spiral's rotations. Conjugation of the number $A$ establishes a spiral with the same intensity of convolutions, but with the opposite rotation. If the motion of the point in the orbit is clockwise in the first case, then it is counter clockwise in the other (Figure 3). If the number is real, or the real part is zero, then the orbit is a meridian - the point does not rotate. The orbit of the point zero is a single point - zero. A vortex is actually a product of exponential growth $t \in \mathbb{R} \rightarrow \rho^{t} \in \mathbb{R}$ and of rotation of the circle $t \in \mathbb{R} \rightarrow e^{i t} \in \mathbb{C}$. A vortex is motion $(t, \psi) \in \mathbb{R} \times[0,2 \pi] \rightarrow \rho^{t} e^{(\phi t+\psi) i}$, where $A=\rho e^{i \phi}$ and $\rho^{2}=a^{2}+b^{2}$.

\section{Spirals on a sphere}

The field of complex numbers $\mathbb{C}$ compactified by one point becomes the two-dimensional sphere, the added point is called the North Pole $\infty$, the point zero is called the South Pole 0 , and the group $\mathbb{T}$ becomes the Equator of this sphere. For the sake of the subsequent inquiry, it is convenient to assume that $0 \cdot z=0, \infty \cdot z=\infty$, where $z$ is any point of the sphere. As a consequence, the product of zero and infinity is not commutative, because it holds $0 \cdot \infty=0$, but $\infty \cdot 0=\infty$ The southern hemisphere can be represented parametrically in the form $x=r \cos \phi, y=r \sin \phi, z_{s}=\theta$, where $r \in[0,1]$, $\phi \in[0,2 \pi)$; and parameter $\theta$ satisfies $(\rho-1)^{2}+r^{2}=1$, so $\theta=1-\sqrt{1-r^{2}}$. The equation of the northern hemisphere is given by analogous formulas: $x=r \cos \phi, y=r \sin \phi, z_{N}=2-z_{S}$, where $r \in[0,1], \phi \in[0,2 \pi)$. Therefore, the North Pole has the coordinates $(0,0,2)$, and the South Pole $(0,0,0)$ Complex numbers are made equivalent to a sphere with the radius 1 and the 
center $(0,0,1)$, naturally exclusive of the north pole. After compactification, a field $\mathbb{C}$ develops into a sphere represented by the equation $x^{2}+y^{2}+(z-1)^{2}=1$. A parametric representation of a spiral on this sphere has the form:

$$
\left\{\begin{array}{l}
x=\rho^{-|t|} \cos (\phi t+\psi) \\
y=\rho^{-|t|} \sin (\phi t+\psi) \\
z=1+\operatorname{sing}(t) \sqrt{1-\rho^{-2|t|}}
\end{array}\right.
$$

where $\rho$ is the modulus of the complex number $A=\rho e^{i \phi}=a+b i, \phi-$ the angle generated by this number, $\psi$ - the angle related to the number $u \in \mathbb{T}$ and $t \in \mathbb{R}$. If $\rho>1$, then a vortex is the outflow from the South Pole and moves northbound, whereas if $\rho=1$ then a vortex is a stable rotation whose orbits are parallels (circles of latitude). If $\rho<1$, then a vortex moves southbound from the north -the point zero, i.e. the South Pole is the equilibrium point. For $\rho \neq 1$ poles are skew-symmetric points: when a point on the orbit moves about one pole to the left, then its motion around the other pole is in the opposite direction. The poles are the limit points of these orbits (Figure 4).
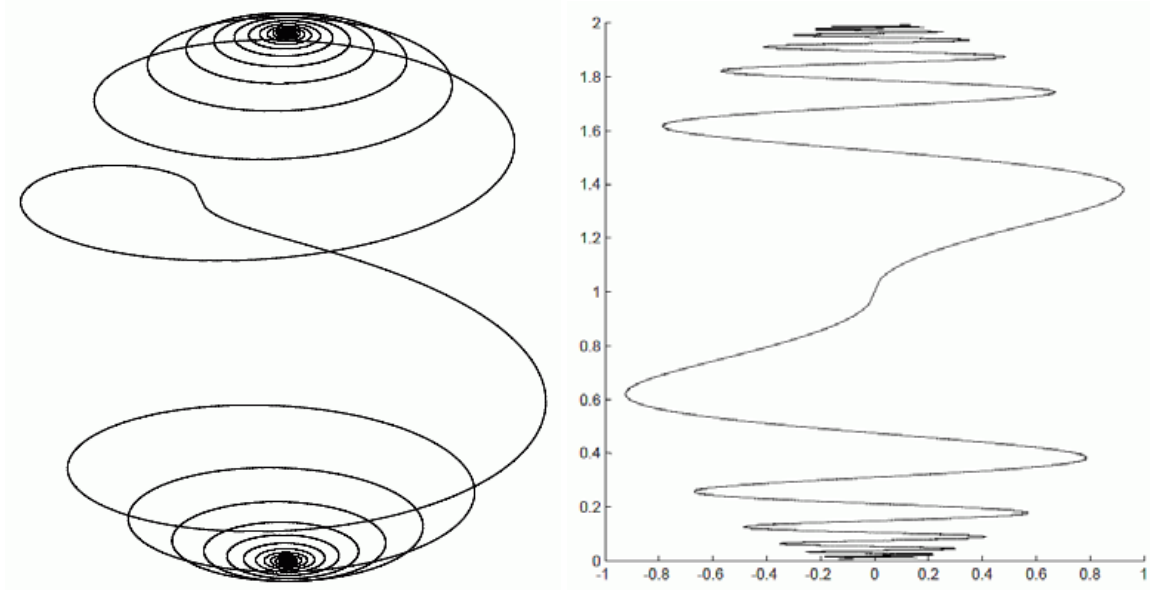

Fig. 4. A spiral on a cone and its graphical projection on the XZ plane

Source: own elaboration. 
One may otherwise explain that complex numbers dance around poles. The following kinds of orbits are distinguished: clockwise and counterclockwise spiral orbits, meridian (semicircular) orbits and parallels. Parallel orbits separate the set of clockwise spiral orbits from the set of counterclockwise spiral orbits, whereas meridian orbits separate the set of spiral orbits of complex numbers with the strictly negative imaginary part from the set of spiral orbits of complex numbers with the strictly positive imaginary part. A family of spiral orbits of the corresponding type is stable. On the other hand, families of parallel and meridian orbits are not stable - they can be regarded as catastrophic states, because any trivial shift in the number generating a vortex induces a completely different family of orbits.

Using the above mentioned cogs $\mathbb{T}_{n}$, let us introduce a general definition of a periodic function. Periodicity is related to automorphic functions. If $G$ is any group, and $H-$ a normal subgroup $G$, then the function $f: G \rightarrow Y$ is periodic with respect to a subgroup $H$, if $f(x)=f(x+h)$, in an additive notation, for $x \in G, h \in H$. Thus, the function $f$ is automorphic with respect to a subgroup $H$. Its translations remain unchanged. A group $\mathbb{T}$ is a set of objects called transpositions in elementary teaching. The quotient group $P_{1}(\mathbb{R})=\mathbb{T} / D$, where $D$ is a two-element group $\{-1,1\}$, is a family of directions on a plane. What are the continuous functions from a linear space $C\left(P_{1}(\mathbb{R}), \mathbb{R}\right)$ ? They are periodic continuous functions belonging to a space of continuous functions $C(T, \mathbb{R})$, whose diagrams are symmetric with respect to any plane passing through the axis of the cylinder in which these diagrams are positioned. The existence of the group $P_{1}(\mathbb{R})$ is obvious in terms of algebra. However, this is a topological group. Is it possible to attach opposite points of the circle so as to change the direction of rotation? In practice it is not possible - there is no model of such a group in nature. It is worth noting besides that $P_{1}(\mathbb{R})=\mathbb{T} / D=\mathbb{C}^{*} / \mathbb{R}^{*}$ and $D=\mathbb{R}^{*} / \mathbb{R}_{+}^{*}$. A topology on $P_{1}(\mathbb{R})$ is the strongest topology in which a projection $\Psi: T \rightarrow P_{1}(\mathbb{R})$ defined by the formula $\Psi(u)=u$ / $D$ is still a continuous function.

The real time is a mixture of circular time with linear time. Such a combination yields a spiral on a cone. If the motion along the spiral takes place to the top of the cone, then we deal with a stabilizing vortex - a system given by the spiral tends to an equilibrium. If the motion is in the opposite direction, away from the top of the cone, then a cybernetic system de- 
fined by such a spiral becomes destabilized and as a consequence it is destroyed. The equation of an elliptical cone is of the form: $\frac{x^{2}}{a^{2}}+\frac{y^{2}}{b^{2}}-\frac{z^{2}}{c^{2}}=0$; if $a=b$, then the cone is rotational - circular. A parametrical representation of this cone is given by the system of equations:

$$
\left\{\begin{array}{c}
x=a t \cos \phi \\
y=b t \sin \phi \\
z=c t
\end{array},\right.
$$

where $t, \phi \in \mathbb{R}$. So a spiral on this cone is a line given parametrically by the system of equations:

$$
\left\{\begin{array}{l}
x=a t \cos (\tau t+\psi) \\
y=b t \sin (\tau t+\psi) \\
z=c t
\end{array}\right.
$$

where $t \in \mathbb{R}$, and the quantities $a, b, c, \tau, \psi \in \mathbb{R}$ are fixed. In addition, we recall that Archimedes' spiral in polar coordinates is defined by the equation $r=\rho \phi$, where $\phi \in \mathbb{R}$.

The observed stock exchange data make it possible to identify a cone and to determine a spiral that describes a stock market performance. The estimated cone is presented in a canonical form, that is given above, suitably changing the coordinates. The canonical form provides a timely recognition of market trends: whether a stock exchange tends to equilibrium or to destabilization. Stock exchange data are typically observed as a curve with vertices (a polygonal chain) that is equivalent to a projection of a spiral placed in a cone onto a plane. Certainly, a character of this spiral varies with time. Stock exchange time can be naturally divided into homogenous intervals in which a spiral remains stable. The type of the spiral can be an indication for investors. If the examined system tends to destabilization, then one can either earn a lot or lose a lot; when the system tends to stabilization, risks are reduced, but profits are moderate.

\section{Practical remarks}

A vortex is measured by a vortex and length by length. There exist vortices, perhaps not in nature, but only in theory, which cannot be measured 
by complex numbers, in a similar way as there exist artificial lines without lengths. The richness of complex numbers supports the hypothesis that one can measure any natural vortex by an appropriate complex number.

Measuring a vortex observed on a windy day is a straightforward activity. One takes two pictures of whirling dust so as to determine the three points: the center $s$ of the vortex, the initial point $p$ and its image $q$. A ratio of the distance $r_{1}=\overrightarrow{s p}$ to the distance $r_{2}=\overrightarrow{s q}$ is the modulus of a complex number, and the angle between its radiuses $\overrightarrow{s p}, \overrightarrow{s q}$ and its argument $\phi$. Therefore $A=\rho e^{i \phi}$.

A cobweb model for determining the equilibrium price is a well-known economic equivalent of a vortex. If the relationship between supply and price and the relationship between demand and price are known, then a cobweb model defines a price equate at which supply and demand are balanced [Jakimowicz, Juzwiszyn 2012].

Galloping inflation is an example of a harmful phenomenon that results in motion leading to destabilization - excess money supply in the market causes a financial crisis marked by a general price increase [Dechert 1996]. Typical vortices are observed on a stock exchange. These are spiral motions on a cone resulting from changes in numbers of stocks and their prices over time [Juzwiszyn 2010]. Vortices do not exist without time, as they imply a change and motion. In order to describe an economic vortex by a single complex number, we need to measure it. Vortices concerning stock exchanges should be measured in short time intervals. A measurement consists in determining an angle and a radius of a vortex, i.e. a related complex number is determined. The case in outer space is similar, however the measurements are carried out in appropriately long time intervals.

The rotational motion commonly observed in outer space results from gravitation forces among material objects. Two bodies independently sent into space will establish one set of objects rotating around each other after some time. Every day we deal with a sun clock containing the three cogs the Sun, the Earth and the Moon. The music of the celestial spheres is an ancient concept. The music of celestial and financial spheres is encoded by complex numbers. The sound of these numbers is an open question.

The frequent turbulences observed in nature and in societies result from interactions among different forces. The net force is a flat spiral or a spatial spiral on a cone. Social disturbances stimulated by agitators can turn into anarchy and destroy the entire society. Therefore, political, religious and professional groupings should be monitored. Social organizations are con- 
structive when they unite the community by stronger bonds between individuals. The most healthy and generally regarded as good, are societies in which a network of traditional linkages attracts most people. Differences of opinions in such society, if any, always lead to stabilization, and often increase the stability of the whole by generating new bonds. A program uniting the society in a self-defense against global threats is known in the Netherlands as the polder model. The idea to mitigate social differences was born there in the $16^{\text {th }}$ century after a major flood that destroyed about one third of the country. Polders, land reclaimed from the sea, are protected by slopes, dams and various kinds of dykes. An excess of water is pumped out by windmills all the time onto a higher level. Care to maintain the protection system is crucial for all the inhabitants of the regions always threatened by the sea. An individual concern must be subject to the common interest in every respect. It is a battle against the forces of nature and the principles of war apply. One has to endure on duty till the end. A legend says that a little Dutch boy saw a leaking dike, put his finger into the hole, staying there all night, and was found dead on duty. Water did not flood the polder [Smoluk 2002; Mc Nutt 2002].

Strikes of various groups of employees and political actions by socially radical groups produce dangerous social turbulences. A good example of a model resembling the Dutch polder model applied to solve a social crisis occurred in ancient Rome when plebeians revolted against patricians. Menenius Agrippa, the consul of the Roman Republic ( $6^{\text {th }} / 5^{\text {th }}$ century BC), told a clever fable about the parts of the human body rebelling against the stomach. The plebeians were persuaded that the whole can be destroyed and eventually mitigated their anger.

No social group may live at an expense of other groups, otherwise the whole community grows weaker and ailing. Odi et amo - I hate and I love. Hatred destroys and destabilizes - love unites and builds. Yet, if you want to be loved, you have to love as well - si vis amari, ama. The three ancient social groups stem from the three sons of Noah: Shem, Ham and Japheth. Tu Shem ora, Ham labora, Japheth rege at protege. Medieval society was a pyramid with noblemen and priests on its top, knights as its core and peasants at the bottom. A social ladder stood on the back of a labourer, was supported by a knight and crowned with a priest.

Complex numbers play a part here in a most unusual way, as they provide both a beautiful and a unique tool for describing both social movements and the Earth's atmosphere as well as a planetary system and the whole universe. 


\section{References}

Dechert W.D. (ed.). (1996). Chaos Theory in Economics: Methods, Models and Evidence. Edward Elgar Publishing. Cheltenham.

Jakimowicz A., Juzwiszyn J. (2012). Vortex stabilization of market equilibrium in theory and in practice of economics. Acta Physica Polonica A 121(2B). Pp. 54-60.

Juzwiszyn J. (2010). Verified assumptions of the economic theory of whirlpools. Mathematical Economics 6 (13). Pp. 49-64.

Mc Nutt P.A. (2002). The Economics of Public Choice. Edward Elgar Publishing Limited. Cheltenham (UK).

Smoluk A. (2002). Co jest przedmiotem rachunku prawdopodobieństwa [What is the object of probability theory]. Ekonomia Matematyczna 6. Pp. 27-48. 